\title{
Quasi-linear theory of Cherenkov-drift instability: Application for radio pulsars
}

\author{
D. Shapakidze ${ }^{1, \star}$, G. Melikidze ${ }^{1}$, and G. Machabeli ${ }^{2}$ \\ 1 Institute of Astronomy, University of Zielona Góra, Lubuska 2, 65-265 Zielona Góra, Poland \\ ${ }^{2}$ Center for Plasma Astrophysics, Abastumani Astrophysical Observatory, Al.Kazbegi ave. 2a, Tbilisi 380060, Georgia
}

Received 5 June 2002 / Accepted 4 July 2002

\begin{abstract}
We present a plasma model for formation of discontinuous position-angle curves observed in some radio pulsar profiles. The model is based on the polarization properties of radio waves excited by Cherenkov-drift instability developing in pulsar magnetospheres. The radiation is confined in a cone of emission and consists of two orthogonally polarized waves. Examination of a quasi-linear stage of Cherenkov-drift instability shows that the excitation mechanism provides the modes with different spectral energy densities depending on the direction of $\boldsymbol{k}$. As a pulsar rotates, an observer's sight-line track crosses different mode-predominated areas on the base of the emission cone. Transition of the sight-line track from one mode-predominated area to another results in the formation of pulsar polarization fluctuations observed in polarization angle distribution displays and average waveforms.
\end{abstract}

Key words. stars: pulsars: general - polarization - radiation mechanisms: non-thermal - plasmas - instabilities

\section{Introduction}

We propose a plasma mechanism to explain the nature of discontinuities observed in polarization position-angle (PA) sweeps plotted on average pulse profiles of some radio pulsars (Stinebring et al. 1984a; Gil \& Lyne 1995; Asgekar \& Deshpande 2000). PA curves usually show a smooth "S-shaped" variation across the pulse profile, which is theoretically well interpreted in the framework of the rotating vector model (Radhakrishnan \& Cooke 1969). However, as yet, there is no satisfactory explanation of observed discontinuous jumps (almost equal to $90^{\circ}$ ) of the PA curve instead of the smooth S-shaped trace. The displays of average-pulse profiles of such pulsars (Backer \& Rankin 1980; Stinebring et al. 1984a, 1984b) left no doubt that the PA curve discontinuities are due to the presence of orthogonally polarized modes in pulsar emission. It was supposed that the different orthogonal modes are dominant for different parts of these profiles and, so, instead of the smooth S-shaped curve of PA, there is a discontinuous step in the curve (Lyne \& Graham-Smith 1998).

In this paper we consider the plasma mechanism for generation of the orthogonally polarized radio emission from pulsars and study plasma energy redistribution between the orthogonal modes. Our mechanism is intrinsic to the plasma model

Send offprint requests to: D. Shapakidze,

e-mail: shapo@ia.uz.zgora.pl

* Also at Center for Plasma Astrophysics, Abastumani Astrophysical Observatory, Tbilisi, Georgia. of pulsar radio emission elaborated in the series of works by Kazbegi et al. (KMM) (1989, 1991a, 1991b); Kazbegi et al. $(1989,1991)$ and also by Lyutikov et al. (1999).

In the framework of the plasma model, pulsar radiation can be generated mainly by two types of plasma instabilities - by the cyclotron instability and the so-called Cherenkovdrift instability (ChDI). ChDI excites two orthogonally polarized plasma modes. These are transverse $(t)$ and longitudinaltransverse (lt) waves. Applying the method used to treat the quasi-linear theory of cyclotron instability (Lominadze et al. 1979, 1983; Lyutikov 1998; Malov \& Machabeli 2001), we examine the quasi-linear stage of ChDI and derive the ratio between the spectral energy densities of these waves, $W(\boldsymbol{k})$. As we demonstrate in Sect. 2, the ratio depends on the direction of the wave propagation. This means that the waves of different polarization predominate in the adjacent areas of a conical emission beam. So, the transition of the observer's line of sight from one area to another should be accompanied by a sharp change of observed polarization and corresponding jumps in the PA curve of almost in $90^{\circ}$.

The organization of the paper is as follows. First, in Sect. 2 we discuss the development of $\mathrm{ChDI}$ in pulsar magnetospheres taking into account the curvature drift of the resonant particles and study a quasi-linear relaxation of the instability. In Sect. 3 we propose a model for explanation of the nature of discontinuities observed in polarization position-angle (PA) sweeps and finally, in Sect. 4, we offer a summary of our results. 


\section{Cherenkov-drift instability}

The necessary condition for the development of Cherenkovdrift instability (as for usual Cherenkov instability) is the presence of a beam of particles in plasma. The plasma of pulsar magnetospheres is assumed to be a steady, space charge separated limited flow along the open curved magnetic field lines (Arons 1981; Muslimov \& Tsygan 1992). It consists of i) a bulk of secondary plasma particles, emanated with low Lorentz factors $\gamma_{\mathrm{p}} \sim 3 \div 10$ (Machabeli \& Usov 1989), and ii) a beam of primary particles (electrons or ions, depending on the magnetic dipole orientation) ejected from the stellar surface with Lorentz factors $\gamma_{\mathrm{b}} \sim 10^{6} \div 10^{7}$. ChDI develops when the beam particle curvature drift is significant. The drift velocity is directed across the plane of magnetic field lines curvature and is:

$u_{\mathrm{d}}=\frac{\gamma v_{\|}^{2}}{\omega_{B} R_{B}}$.

Here $\omega_{B}=q B / m c$ is the cyclotron frequency of the particle of charge $q$ and mass $m ; c$ is the speed of light; $R_{B}$ is the curvature radius of the magnetic field line; $\gamma$ is the Lorentz factor of the particle and $v_{\|}$is a component of the particle velocity $(\boldsymbol{v})$ along the magnetic field line. If $\gamma \gg 1$, the value of the drift velocity $u_{\mathrm{d}}$ can be significant. Let us note that electrons and ions are drifting in opposite directions.

The waves are generated when the Cherenkov-drift resonance condition fulfills:

$\omega-k_{\|} v_{\|}-k_{x} u_{\mathrm{d}}=0$,

where the $x$-axis is directed transversely to the plane of the curved magnetic field lines. The positive direction of $x$-axis is chosen toward the drift of electrons.

One of the excited waves is a transverse $t$-wave, the electric field of which is directed perpendicularly to the plane of the wave vector and the external magnetic field $\left(\boldsymbol{k}, \boldsymbol{B}_{0}\right)$. The other one is a longitudinal-transverse $l t$-wave. Its electric field vector lies in the $\left(\boldsymbol{k}, \boldsymbol{B}_{0}\right)$ plane. So, $t$ and $l t$ waves, propagating in the same direction, are orthogonally polarized. The growth rate of these waves is written as:

$\Gamma_{k}=\frac{\pi}{2} \frac{\omega_{\mathrm{b}}^{2}}{\omega} \frac{\gamma_{\mathrm{b}}}{\gamma_{\mathrm{T}}^{2}} A_{k}$

and reaches maximum when

$\omega=\frac{\omega_{\mathrm{b}} \gamma_{\mathrm{b}} c}{\gamma_{\mathrm{T}}^{3 / 2} u_{\mathrm{d}}}$

Here $A_{k}=\left(k_{r} / k_{\perp}\right)^{2}$ for $t$-waves and $A_{k}=\left(k_{x} / k_{\perp}\right)^{2}$ for $l t$-waves; $\gamma_{\mathrm{b}}, \omega_{\mathrm{b}}$ and $\gamma_{\mathrm{T}}$ are Lorentz factors, Langmuir frequency and thermal spread of the beam, respectively (KMM 1989, 1992 and Kazbegi et. al 1991). The excited waves are confined in a cone of emission with a small opening angle $\vartheta_{0} \approx u_{\mathrm{d}} / c$.

Studying the back reaction of the excited waves upon the resonant particles is the next step of investigation of ChDI. We treat the problem in a quasi-linear approximation (see Shapakidze et al. 2002), when the energies of $t$ and $l t$ modes, $W=\int W(\boldsymbol{k}) \mathrm{d} \boldsymbol{k}$, are still much less than the energy of plasma particles, $W \ll W_{\mathrm{p}}$. The development of ChDI results in the diffusion (in the momenta space) of the resonant particles of a beam. The diffusion occurs along as well as across the magnetic field lines. The expressions for diffusion coefficients along $\left(D_{||||}\right)$as well as across $\left(D_{\perp \perp}\right)$ the magnetic field lines are derived by Shapakidze et al. (2002).

The quasi-linear relaxation of ChDI is described by the following equation:

$$
\begin{aligned}
\frac{\partial f_{0}}{\partial t}= & \frac{\partial}{\partial p_{\|}}\left(D_{\|||} \frac{\partial f_{0}}{\partial p_{\|}}-F_{\|} \frac{f_{0}}{m c}\right) \\
& +\frac{1}{p_{\perp}} \frac{\partial}{\partial p_{\perp}} p_{\perp}\left(D_{\perp \perp} \frac{\partial f_{0}}{\partial p_{\perp}}-F_{\perp} \frac{f_{0}}{m c}\right) .
\end{aligned}
$$

Here $p_{\|}$and $p_{\perp}$ are the components of particle dimensionless momenta $(\boldsymbol{p}=\gamma \boldsymbol{v} / c)$ along and across the external magnetic field, respectively. In Eq. (5) we consider action of the force $\boldsymbol{F}$ on a relativistic particle spiralling along the inhomogeneous (dipolar) magnetic field. $\boldsymbol{F}=\boldsymbol{F}_{R}+\boldsymbol{F}_{B}$, where $\boldsymbol{F}_{R}$ is the force of radiation-induced particle deceleration:

$\left(F_{R}\right)_{\|}=-\frac{2}{3} \frac{q^{2} \omega_{B}^{2}}{c^{2}} \gamma^{2} \psi^{2} ; \quad\left(F_{R}\right)_{\perp}=-\frac{2}{3} \frac{q^{2} \omega_{B}^{2}}{c^{2}} \gamma^{2} \psi^{3}$

and $\boldsymbol{F}_{B}$ is the force due to the conservation of the adiabatic invariant $p_{\perp}^{2} / B_{0}=$ const. (Lominadze et al. 1979; Lominadze et al. 1983; Lyutikov 1998):

$\left(F_{B}\right)_{\|} \simeq \frac{m c^{2}}{R_{B}} \gamma \psi^{2} ; \quad\left(F_{B}\right)_{\perp} \simeq-\frac{m c^{2}}{R_{B}} \gamma \psi$.

Here $\psi \approx p_{\perp} / p_{\|}$is the pitch angle of the resonant particle.

A stationary state $\left(\partial f_{0} / \partial t=0\right)$ may be reached when the both righthand terms in Eq. (5) are equal to zero or cancel each other. As for the transverse diffusion, it is balanced by the perpendicular forces. To estimate the relative importance of the parallel quasi-linear diffusion and $F_{\|}$we have to consider the ratio between the terms $D_{\|\mid\|}\left(\partial f_{0} / \partial p_{\|}\right)$and $F_{\|}\left(f_{0} / m c\right)$ in Eq. (5). Estimations show that for the parameters of a typical pulsar magnetosphere, the contribution of the parallel diffusion significantly exceeds the contribution of the parallel forces:

$\frac{m c D_{|\||}}{\gamma\left(F_{R}\right)_{\|}}=1.5 \times 10^{4} \frac{\gamma_{6} P_{0}^{3 / 4} r^{3 / 2}}{\dot{P}_{-15}^{1 / 4} \psi_{-4}^{2}}$,
$\frac{m c D_{\|\| \mid}}{\gamma\left(F_{B}\right)_{\|}}=1.3 \times 10^{4} \frac{P_{0}^{19 / 4} r^{13 / 2}}{\dot{P}_{-15}^{5 / 4} \psi_{-4}^{2}}$.

Here we have used the simplified expression of $D_{||||}$(see Shapakidze et al. 2002):

$D_{\text {||I| }} \simeq 8 \pi U^{2}\left(\frac{q}{m c}\right)^{2} \frac{W}{\Gamma}$,

where $U=u_{\mathrm{d}} / c$ for $t$-waves and $U=\left(u_{\mathrm{d}} / c+k_{x} / k_{\|}\right)$for $l t$ waves. In our estimations, the values of all parameters are referred to the plasma model of pulsar radiation. The model suggests that the instability develops at the distances $R \approx 0.5 R_{\mathrm{LC}} \div$ $0.8 R_{\mathrm{LC}}$ from the pulsar, where $R_{\mathrm{LC}}=P c / 2 \pi$ is the light cylinder radius. $P_{0}=P /(1 \mathrm{~s}), \dot{P}_{-15}=\dot{P} / 10^{-15}, \gamma_{6}=\gamma_{\mathrm{b}} / 10^{6}$ and $\psi_{-4}=\psi / 10^{-4}$ are the normalized values of the pulsar rotation period, period derivative, Lorentz factor and the pitch angle of primary beam particles, respectively (it is assumed that 
$P=1 \mathrm{~s}$ and $\dot{P} \sim 10^{-15}$ for typical pulsars; $\psi \sim 10^{-5} \div 10^{-4}$ ). In the calculations we use the dimensionless distance in units of $0.5 R_{\mathrm{LC}}$, so $r \equiv 2 R / R_{\mathrm{LC}}$. The growth rate of the instability is about $\Gamma \approx 6 \times 10^{2} \div 10^{3} \mathrm{~s}^{-1}$ (assuming $A_{k} \approx 1$ and $\gamma_{\mathrm{T}} \approx 10^{4}$ in Eq. (3)). We consider that the wave energy is about $W \sim 0.01 W_{\mathrm{p}}$. Here $W_{\mathrm{p}} \equiv m c^{2} n_{\mathrm{p}} \gamma_{\mathrm{p}}$, where $n_{\mathrm{p}}$ is the number density of the secondary plasma. The energy equilibrium among the plasma components implies $2 n_{\mathrm{p}} \gamma_{\mathrm{p}} \approx n_{\mathrm{b}} \gamma_{\mathrm{b}}$, where $n_{\mathrm{b}}=B_{0} / P c q$ is the number density of the primary beam. The external magnetic field is dipolar: $B_{0}=B_{\mathrm{S}}\left(R_{\mathrm{S}} / R\right)^{3}$, where $B_{\mathrm{s}} \approx 3.2 \times 10^{19}(P \dot{P})^{0.5} \mathrm{G}$ is the magnetic field at the surface of a pulsar. Finally, using Eq. (1) we can estimate the value of the drift velocity as $u_{\mathrm{d}} / c \approx 2.6 \times 10^{-2} \gamma_{6} P_{0}^{2.5} \dot{P}_{-15}^{-0.5} r^{3}$.

Keeping in mind that the parallel forces contribute to the quasi-linear relaxation insignificantly, we write down the quasi-linear equation for the distribution function as follows:

$\frac{\partial}{\partial t} f_{0}\left(p_{\|}, t\right)=\frac{\partial}{\partial p_{\|}} D_{\|\|} \frac{\partial}{\partial p_{\|}} f_{0}\left(p_{\|}, t\right)$.

It is easy to find out that the diffusion coefficients, in some sense, define the rate of alteration of $f_{0}$, in the same way as the growth rate defines the wave amplification:

$\frac{\partial}{\partial t} W(\boldsymbol{k}, t)=2 \Gamma_{\boldsymbol{k}} W(\boldsymbol{k}, t)$.

According to the standard procedure, the set of Eqs. (10) and (11) yields a quasi-linear integral of the instability:

$\frac{\partial}{\partial t}\left(f_{0}-\frac{\partial}{\partial p_{\|}} U^{2} \int_{-\infty}^{\infty} \mathcal{R}(\boldsymbol{k}, t) \mathrm{d} \boldsymbol{k}\right)=0$,

where $\mathcal{R}(\boldsymbol{k}, t) \equiv(2 / \pi)\left(\omega / \Gamma_{\boldsymbol{k}}\right)\left(W(\boldsymbol{k}, t) / W_{\mathrm{p}}\right)$. In our calculations we model the initial distribution function of the beam as

$f_{0}\left(p_{\|}, 0\right) \sim \frac{1}{\gamma_{\mathrm{T}}} \exp \left[-\frac{\left(p_{\mathrm{b}}-p_{\|}\right)^{2}}{\gamma_{\mathrm{T}}^{2}}\right]$

( $p_{\mathrm{b}}$ is a mean momentum of the beam particles) and make the following estimation:

$\frac{\partial}{\partial p_{\|}} f_{0}\left(p_{\|}=p_{0}, 0\right) \sim\left(\frac{1}{\gamma_{\mathrm{T}}}\right)^{2}$

(here, $p_{0}$ is a momentum of the resonant particles and $\gamma_{\mathrm{T}} \approx$ $\left.p_{\mathrm{b}}-p_{0}\right)$.

Let us assume that the quasi-linear reaction of both $t$ and $l t$ waves change the distribution function in the same way. So, we can substitute the following equation for Eq. (12):

$$
\begin{aligned}
\Delta f_{0} & =\frac{\partial}{\partial p_{\|}}\left(\frac{u_{\mathrm{d}}}{c}\right)^{2} \int_{-\infty}^{\infty} \mathcal{R}_{t}(\boldsymbol{k}, \infty) \mathrm{d} \boldsymbol{k} \\
& =\frac{\partial}{\partial p_{\|}}\left(\frac{u_{\mathrm{d}}}{c}+\frac{k_{x}}{k_{\|}}\right)^{2} \int_{-\infty}^{\infty} \mathcal{R}_{l t}(\boldsymbol{k}, \infty) \mathrm{d} \boldsymbol{k},
\end{aligned}
$$

where $\Delta f_{0} \equiv f_{0}\left(p_{\|}, \infty\right)-f_{0}\left(p_{\|}, 0\right)$ is an alteration of the distribution function. It is taken into account that the energy of the waves initially equals zero, $W(\boldsymbol{k}, 0)=0$, and consequently $\mathcal{R}(\boldsymbol{k}, 0)=0$ as well.
From Eq. (15) we easily express the ratio between the energies of $t$ and $l t$ waves by means of transverse components of the wave vector $\left(k_{x}, k_{r}\right)$ :

$$
\frac{W_{t}(\boldsymbol{k}, \infty)}{W_{l t}(\boldsymbol{k}, \infty)}=\left(1+\frac{k_{x} c}{k_{\|} u_{\mathrm{d}}}\right)^{2}\left(\frac{k_{r}}{k_{x}}\right)^{2}
$$

In the following section we attempt to apply our result to explain the formation of discontinuities of PA curves.

\section{Polarization of pulsar radio emission generated by Cherenkov-drift instability}

Cherenkov-drift radiation is confined within the emission cone of a small opening angle $\vartheta_{0}$. The radiation is generated in the rotating pulsar magnetosphere and detected by an observer while its line of sight is crossing the base of the emission cone. Transverse components of the wave vector $\left(k_{x}, k_{r}\right)$ define the observed area of the base. On the other hand, they also define the mode with predominant intensity (see Eq. (16)). Consequently, we conclude that in our model the pulsar emission occurs in one orthogonal mode or the other (i.e. an observer should identify the $t$ or $l t$ waves of different polarization) while passing through the different areas on the base of the emission cone. Transition of the observers' line of sight from one area to another should be accompanied by a sharp change in the observed polarization and corresponding jump of the PA curve by $90^{\circ}$.

The area where $t$ mode predominates should be separated from the area of $l t$ mode domination by the lines of the spectral energy equality curve. It is handy to plot a diagram of the energy equality curve - the solution of Eq. (16) in the case $W_{t}(\boldsymbol{k})=W_{l t}(\boldsymbol{k})-$ in polar coordinates $\left(k_{\perp}, \phi\right)$, where $k_{\perp} \cos \phi=k_{x}$ and $k_{\perp} \sin \phi=k_{r}$ :

$k_{\perp}(\phi)= \pm\left(\frac{u_{\mathrm{d}}}{c}\right) \frac{k_{0 \perp}}{\vartheta_{0}} \mathcal{S}_{\mp}(\phi), \mathcal{S}_{\mp}(\phi) \equiv \frac{\cos \phi \mp \sin \phi}{\sin \phi \cos \phi}$.

Here $k_{0 \perp} / \vartheta_{0}$ is substituted for $k_{\|}$in Eq. (16), where $k_{0 \perp}$ is the perpendicular component of the wave vectors lying on the "surface" of the emission cone with an opening angle $\vartheta_{0}$.

In Fig. 1 it is shown that the observer's line of sight (sightline track) crosses the energy equality curve $k_{\perp}(\phi)$ and passes in a sequence through $t$ and $l t$ wave predominated areas. That is why the arcs of PA curve, corresponding to $t$ and $l t$ waves, can be obtained in a sequence along the pulse window. PA itself should spontaneously change its value by $90^{\circ}$ at the pulse longitudes, where the sight-line track intersects the energy equality curve.

It seems that most cases of pulsar polarization fluctuations can be explained in the framework of our model. Generation of $t$ and $l t$ modes by ChDI is supported by evidence in both high and low-frequency observations that the pulsar radiation is orthogonally polarized (Cordes et al. 1978; Backer \& Rankin 1980; Stinebring et al. 1984a and references therein). Two different polarization modes are well defined while studying the PA distribution on the separated mode profiles (see, e.g., Gil et al. 1992). However, the pure orthogonality is presented either in the center or at the wings of the profile and the modes are by 


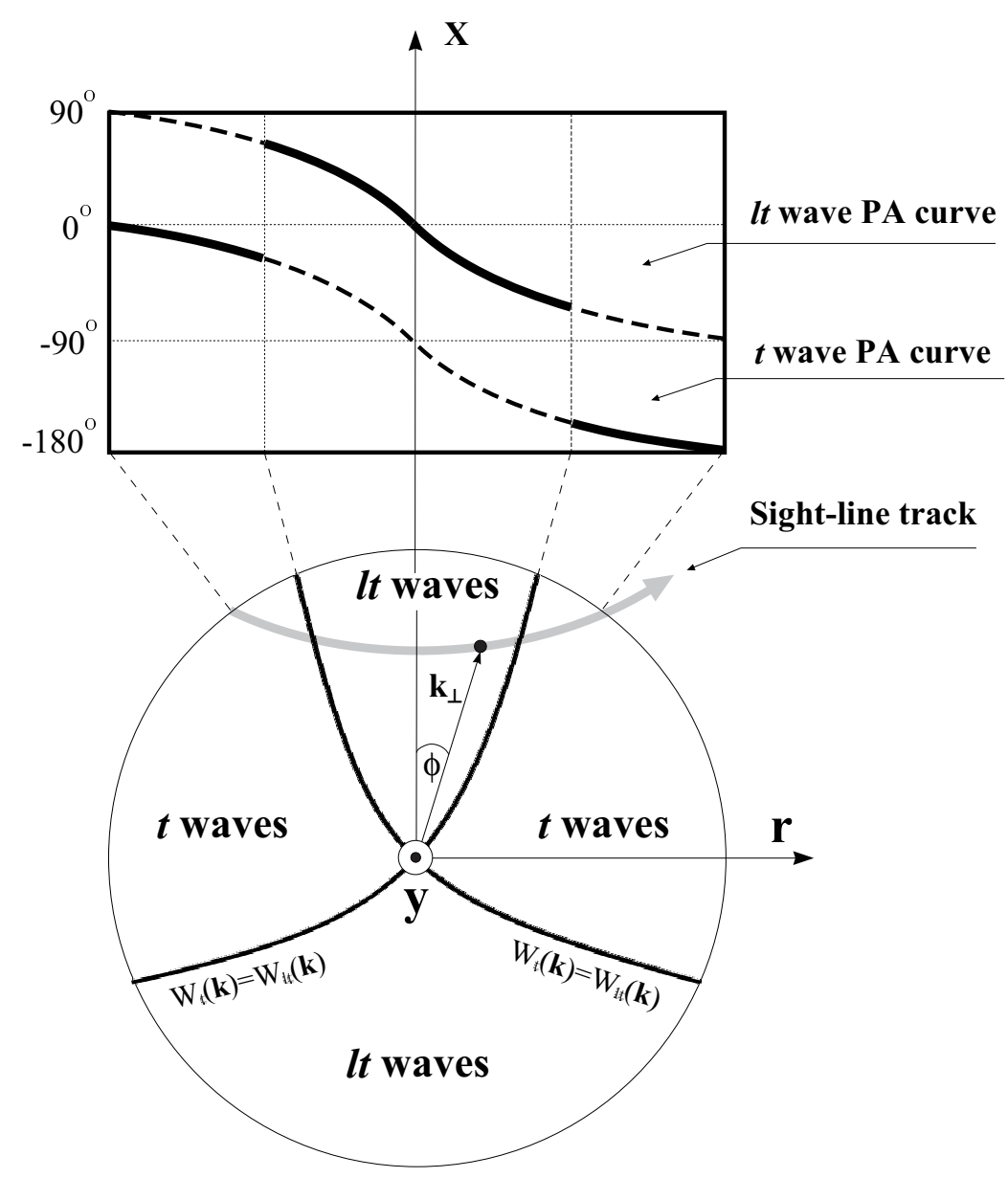

Fig. 1. Formation of orthogonal jumps in the shape of PA curve in the framework of the proposed model. An area, bounded by the large circle, presents the base of emission cone. Here $y$ axis is directed toward us and shows the direction of the magnetic field lines in the wave generation region. Solid curve lines inside the circle represent spectral energy equality lines $\left(W_{t}(\boldsymbol{k})=W_{l t}(\boldsymbol{k})\right)$ of $t$ and $l t$ waves; the gray shaded curve indicates a sight-line track.

no means orthogonal throughout the whole window. Indeed, the deviation from pure orthogonality may be caused by the fact that orientation of the magnetic field $\left(\boldsymbol{B}_{0}\right)$ could be different at the sites of $t$ and $l t$ mode excitation. Hence, $\left(\boldsymbol{k}, \boldsymbol{B}_{0}\right)$ planes could be also differently oriented if modes arrive from different places. Consequently, the angular difference between $\boldsymbol{E}_{t}$ and $\boldsymbol{E}_{l t}$ is not always $90^{\circ}$, but can vary along the pulse window.

The fact that intensities of individual orthogonal modes are not equally distributed within the pulse window (Stinebring et al. 1984a, 1984b; Gil 1987) is well supported by our result (see Eq. (16)). A fine example of the observed intensity distribution within the pulse profile of PSR 0834+06 (Gil 1987, Fig. 3) demonstrates a good fit of our model with observations. It naturally follows from the model that if the sight-line track passes through $t \rightarrow l t \rightarrow t$ waves dominated areas (as it is shown in Fig. 1), then the $l t$ mode should dominate the center of the profile, while the $t$ mode should dominate the wings exactly as in the case of PSR $0834+06$.

A rapid orthogonal jump occurs on the total PA curve at longitudes where modes are of comparable equal intensity (see, e.g., PSR $0329+54$ in Gil \& Lyne 1995). PSR $0329+54$ is one of the best examples of almost orthogonal separation between the modes throughout the whole profile.
The characteristic S shape of the PA curve can be seen in both modes. However, the total PA curve is discontinuous (its shape deviates from the pure S-shaped swing) which clearly indicates the existence of adjacent areas of mode intensity domination. Consequently, we suggest that our model well suits the case of PSR 0329+54 (compare the modelled PA curve in Fig. 1 with that of PSR 0329+54, see Fig. 1 in Gil \& Lyne 1995).

Many profiles of pulsars show the discontinuity of the PA curve at more then one pulse longitude (so-called multidiscontinuity). For example, we can refer to the averaged waveforms of PSR 2020+28 (Stinebring et al. 1984b), PSR J08463533 at $327 \mathrm{MHz}$ and PSR 0834+06 at $35 \mathrm{MHz}$ (Asgekar $\&$ Deshpande 2000). The multi-discontinuity of the shape of the total PA curve can be naturally obtained from our model. Generally, four branches of the energy equality curve compose the solution of $W_{t}(\boldsymbol{k})=W_{l t}(\boldsymbol{k})$ equation. Here the drift velocity $\left(u_{\mathrm{d}} / c\right)$ acts as a scaling factor. Indeed, if the condition $u_{\mathrm{d}} / c>$ $\vartheta_{0} / 2 \sqrt{2}$ is fulfilled, then only two branches of the spectral energy equality curve appear within the base of the emission cone (Fig. 1). If $u_{\mathrm{d}} / c=\vartheta_{0} / 2 \sqrt{2}$, then the same two branches still appear within the base, but two additional branches (passing out of the origin of the reference frame) come closer to the origin and touch the circle delineated by the base of 


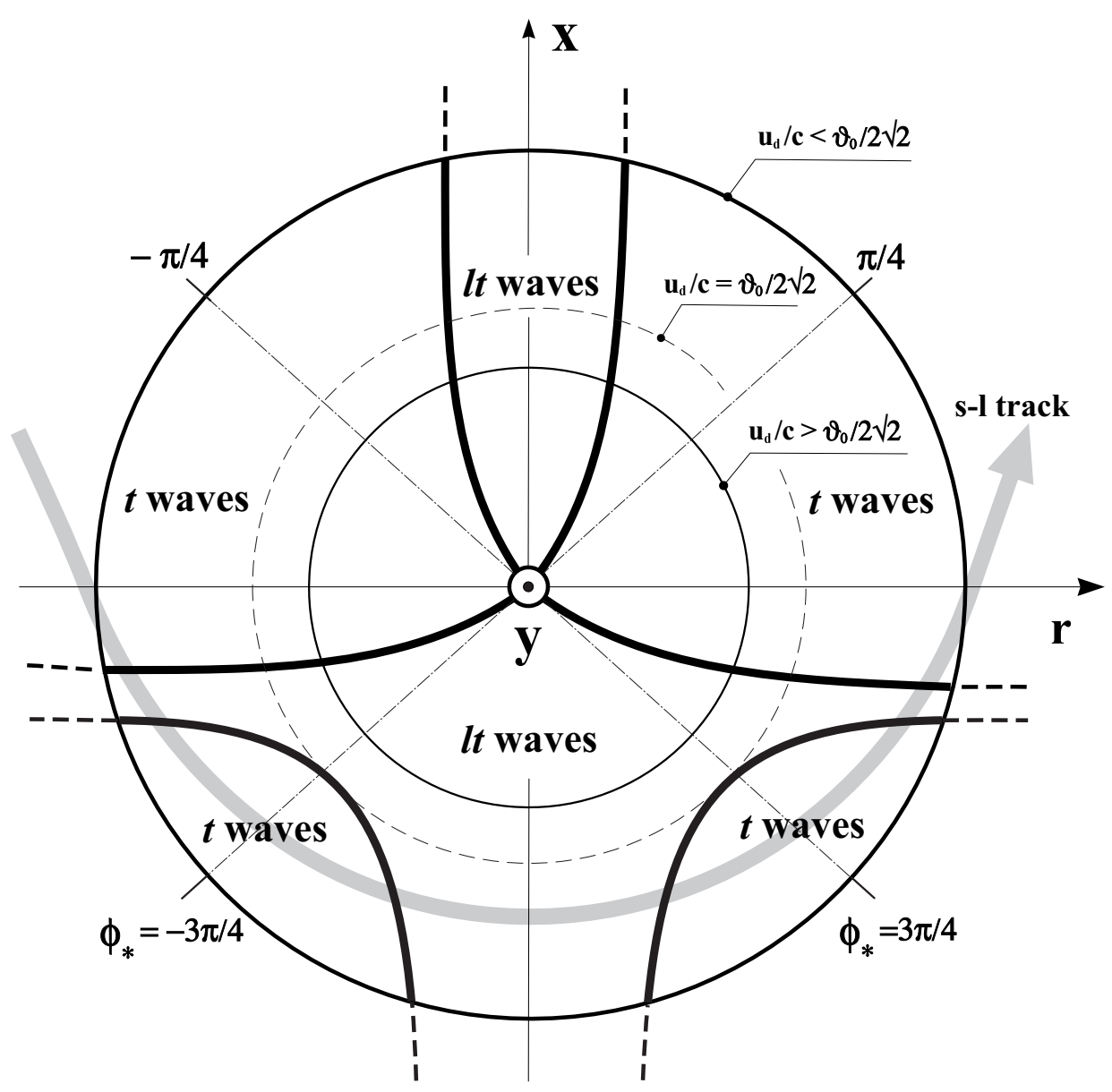

Fig. 2. All four branches of a spectral energy equality curve - the roots of equation $W_{t}(\boldsymbol{k})=W_{l t}(\boldsymbol{k})$ - are plotted in polar coordinates: the lower two branches pass out of the origin of the reference frame, while other two pass trough the origin. Coaxial circles delineate the base of the emission cone for different cases: the inner circle corresponds to the case of $u_{\mathrm{d}} / c>\vartheta_{0} / 2 \sqrt{2}$ (this case is also presented in Fig. 1), when only two branches are located within the base of the emission cone. The large outer circle corresponds to the case of $u_{\mathrm{d}} / c<\vartheta_{0} / 2 \sqrt{2}$, when all four branches are located within the base of emission cone. Two additional $t$-wave predominated areas appeared in this case; the dashed circle corresponds to the case of $u_{\mathrm{d}} / c=\vartheta_{0} / 2 \sqrt{2}$, when lower two branches touch the circle at the angles $\phi_{*}= \pm 3 \pi / 4$; the gray shaded curve indicates a sight-line (s-1) track.

emission cone (dashed circle in Fig. 2). Finally, all four branches appear within the base of emission cone if $u_{\mathrm{d}} / c<$ $\vartheta_{0} / 2 \sqrt{2}$ (Fig. 2). Here $2 \sqrt{2}= \pm \mathcal{S}_{\mp}\left(\phi_{*}\right)$, where $\phi_{*}= \pm 3 \pi / 4+$ $2 \pi n(n=0,1,2, \ldots)$ are the angles at which two additional branches touch the circle. In the case of $u_{\mathrm{d}} / c<\vartheta_{0} / 2 \sqrt{2}$, the sight-line track can cross the energy equality curve several times. That is why a few jumps of PA can be observed.

The various observed waveforms can be modelled by choosing the proper direction of the sight-line tracks relative to the energy equality curves. Indeed, the sight-line track does not necessarily pass the $x$ axis transversely (as it is drawn in Figs. 1 and 2), but could be directed suitably to the given observed waveform (note that sight-line track by definition is transverse to the pulsar rotation axis). That is why our model seems to be flexible concerning the case of multi-discontinuities as well.

\section{Conclusion}

In this paper we present a qualitative model for the formation of discontinuous steps in position angle curves observed in emission of some radio pulsars. The model is based on the polarization properties of plasma waves generated in pulsar magnetospheres due to Cherenkov-drift instability.

In the vicinity of the pulsar light cylinder, two orthogonally polarized, transverse $(t)$ and longitudinal transverse $(l t)$ radio waves can be excited by ChDI. The excitation mechanism provides the modes with different spectral energy densities for different values of the wave vector $\boldsymbol{k}$. Particularly, we find that the ratio of energy spectral densities between $t$ and $l t$ waves is defined by transverse components of the wave vector $\left(k_{x}, k_{r}\right)$. This relation is obtained by studying the quasi-linear stage of ChDI and the ratio depends on the direction of the wave propagation. This means that intensities of individual orthogonal modes are not equally distributed within the base of the emission cone.

Consequently we obtain adjacent areas on the base of the emission cone, where one mode predominates another and vice versa. The areas are separated by the energy equality curves. The sight-line track crosses different mode-predominated areas in a sequence, resulting in the observed variations in the shape of the total PA curve. The discontinuities in the shape of the 
total PA curve should be observed at the longitudes, where the sight-line track intersects the energy equality curve. Most of the cases of pulsar polarization fluctuations could be explained according to the model proposed in this paper.

Acknowledgements. We thank J. Gil for useful discussions. DSh was partially supported by UNESCO Fellowship, International Center for Dense Magnetized Plasma, Institute of Plasma Physics and Laser Microfusion, Warsaw, Poland. The paper is supported in part by KBN Grant 2 P03D 00819 of the Polish State Committee for Scientific Research. We also thank an anonymous referee for useful notes.

\section{References}

Arons, J. 1981, Plasma Astrophys. (European Space Agency, Paris), 273

Asgekar, A., \& Deshpande, A. A. 2000, Pulsar Astronomy, 2000 and Beyond, in IAU Colloq. 177, ed. M. Kramer, N. Wex, \& R. Wielebinski, ASP Conf. Ser. (Sheridan Books, Inc., Michigan), 202, 245

Backer, D. C., \& Rankin, J. M. 1980, ApJS, 42, 143

Cordes, J. M., Rankin, J., \& Backer, D. C. 1978, ApJ, 223, 461

Gil, J. A. 1987, ApJ, 314, 629

Gil, J. A., \& Lyne, A. G. 1995, MNRAS, 276, L55

Gil, J. A., Lyne, A. G., Rankin, J. M., Snakowski, J. K., \& Stinebring, D. R. 1992, A\&A, 255, 181

Kazbegi, A. Z., Machabeli, G. Z., \& Melikidze, G. I. 1989, in Joint Varenna-Abastumani International School \& Workshop on Plasma Astrophysics, ed. T. D. Guyenne (European Space Agency, Paris), 285, 277 (KMM)

Kazbegi, A. Z., Machabeli, G. Z., \& Melikidze, G. I. 1991a, MNRAS, 377 (KMM)
Kazbegi, A. Z., Machabeli, G. Z., \& Melikidze, G. I. 1991b, Aust. J. Phys, 44, 573 (KMM)

Kazbegi, A. Z., Machabeli, G. Z., \& Melikidze, G. I. 1992, Magnetospheric Structure and Emission Mechanisms of Radio Pulsars, in IAU Colloq. 128, ed. T. H. Hankins, J. M. Rankin, \& J. A. Gil (Pedagogical University Press, Zielona Góra, Poland), 373 (KMM)

Kazbegi, A. Z., Machabeli, G. Z., Melikidze, G. I., \& Smirnova, T. V. 1991, Afz, 34, 433 (KMM)

Kazbegi, A. Z., Machabeli, G. Z., Melikidze, G. I., \& Usov, V. V. 1989, in Joint Varenna-Abastumani International School \& Workshop on Plasma Astrophysics, ed. T. D. Guyenne (European Space Agency, Paris), 285, 271

Lominadze, J. G., Machabeli, G. Z., \& Mikhailovskii, A. B. 1979, Sov. J. Plasma Phys., 5, 748

Lominadze, J. G., Machabeli, G. Z., \& Usov, V. V. 1983, Ap\&SS, 90,19

Lyne, A. G., \& Graham-Smith, F. 1998, Pulsar Astronomy (Cambridge University Press, Cambridge), 151

Lyutikov, M. 1998, Phys. Rev. E, 58, 2474

Lyutikov, M., Machabeli, G., \& Blandford, R. 1999, ApJ, 512, 804

Machabeli, G. Z., \& Usov, V. V. 1989, Sov. Astron. Lett., 15, 393

Malov, I. F., \& Machabeli, G. Z. 2001, ApJ, 554, 587

Muslimov, A. G., \& Tsygan, A. I. 1992, MNRAS, 255, 61

Radhakrishnan, V., \& Cooke, D. J. 1969, Astrophys. Lett., 3, 225

Shapakidze, D., Machabeli, G., Melikidze, G., \& Khechinashvili, D. 2002 [astro-ph/0112218], submitted to Phys. Rev.

Stinebring, D. R., Cordes, J. M., Weisberg, J. M., \& Boriakoff, V. 1984a, ApJS, 55, 247

Stinebring, D. R., Cordes, J. M., Weisberg, J. M., \& Boriakoff, V. 1984b, ApJS, 55, 279 